\title{
Professor Ruixia Pei's Experience in Treating Hyperthyroidism
}

\author{
Fen Zhang1, Xingyu Chen1, Di Sun'1, Ruixia Pei²* \\ 1Shaanxi University of Traditional Chinese Medicine, Xianyang 712046, Shaanxi Province, China \\ ${ }^{2} \mathrm{Xi}$ 'an Hospital of Traditional Chinese Medicine, Xi'an 710021, Shaanxi Province, China \\ *Corresponding author: Ruixia Pei, 29prx@163.com
}

\begin{abstract}
In this article, we summarize the clinical experience of Professor Ruixia Pei, a famous traditional Chinese medicine practitioner in Shaanxi Province, China, in treating hyperthyroidism. The etiology and pathogenesis, syndrome differentiation, and medication experience are introduced in detail. This paper summarizes the advantages of Professor Pei's methods of syndrome differentiation and treatment of hyperthyroidism. This may help enrich the clinical treatment of hyperthyroidism, and provide some diagnosis and treatment ideas.
\end{abstract}

Keywords: Hyperthyroidism; Ruixia Pei; Experience; TCM syndrome differentiation treatment

Publication date: July 2021; Online publication: July 30, 2021

\section{Introduction}

Hyperthyroidism is a common endocrine disease caused by increased excitability and metabolic hyperthyroidism due to thyroid itself or various reasons other than thyroid ${ }^{[1]}$. It is characterized by enlargement of both sides of anterior cervical laryngeal node, fear of heat and sweating, excitement and irritability, increased appetite with emaciation, palpitation, and sudden symptoms ${ }^{[2]}$. Compared with Western medicine treatment, traditional Chinese medicine (TCM) treatment allows for comprehensive adjustment ${ }^{[3]}$, and is associated with small-scale side effects and low recurrence rate ${ }^{[4]}$. This form of treatment has gradually been accepted by patients and has achieved certain curative effect in treatment.

Professor Ruixia Pei is the third famous TCM doctor in Shaanxi Province and the first famous TCM doctor in Xi'an city. She has been engaged in clinical and research of endocrine diseases for a long time. In the diagnosis and treatment of thyroid disease, she has accumulated a lot of diagnostic experiences. In this article, we present the experience of Professor Ruixia Pei in the treatment of hyperthyroidism (liver classics fire prosperous type).

\section{Etiology and pathogenesis}

Hyperthyroidism belongs to the category of "gall disease" in TCM [5]: "The one with gall usually has pathogenic fire and turbid $q i$ in the five internal organs, blood stasis and stagnated phlegm rather than any specific disease." It is suggested that gall disease is mainly caused by $q i$ stagnation, phlegm coagulation and blood stasis. Professor Xu Gongping thinks that gall disease is related to qi stagnation, fire hyperactivity, heat toxin, phlegm coagulation and blood stasis, and the main method is to soothe the liver and regulate $q i$, remove phlegm and eliminate gall ${ }^{[6]}$. Professor He Ze thinks that the cause of gall disease is mostly emotional influence, and the pathogenesis is mainly hyperactivity of liver fire, stomach fire and heart fire ${ }^{[7]}$. Based on "treating gall disease from liver," it can be seen that gall disease from liver has been unanimously recognized. 


\section{Disease differentiation and syndrome differentiation}

According to many years of clinical experience, Professor Pei considers liver depression and qi stagnation, and imbalance of $q i$, blood and body fluid are the basic mechanism of pathogenesis, and soothing liver and regulating $q i$, nourishing yin and reducing fire as the general treatment principle.

The main manifestations are palpitation, hand tremors, abnormal sweating, irritability, insomnia, excessive food intake, emaciation, red tongue and yellow fur, and string pulse. For the treatment of clearing liver and relieving fire, regulating $q i$ and resolving phlegm, Longdan Xiegan decoction was used with gentianaceae $6 \mathrm{~g}$, stir-fried gardenia $6 \mathrm{~g}$, bupleurum $10 \mathrm{~g}$, Alisma orientalis $10 \mathrm{~g}$, Scutellaria baicalensis 6 g, Shengdi 15 g, Danshen 20 g, Baishao 15 g, Prunella vulgaris 10 g, etc. The prescription uses Longdan to clear liver fire, Gardenia jasminoides and Scutellaria baicalensis to clear heat and detoxify, Shengdi and Danshen to nourish yin and activate blood, Baishao to soften the liver, Chaihu to soothe the liver to induce various drugs into the liver, and Angelica sinensis and Chuanxiong to activate blood, remove blood stasis and cure insomnia with sour jujube kernel and Rehmannia glutinosa.

\section{Case verification}

A 46-year-old, female patient visited the hospital on August 26, 2020. Her chief complaint was "front neck distension for 3 months and being upset and irritable for 20 days." The patient had no obvious inducement 3 months before the hospital visit and did not pay attention to it. In the past 20 days, she was impatient and irritable, and occasionally had abnormal sweating. She was diagnosed with hyperthyroidism in the local hospital. She was given methimazole tablets $10 \mathrm{mg}$, which were taken orally twice a day. However, the symptoms were not significantly relieved as the patient was still feeling upset and irritable, and had fatigue, distension and discomfort in front of neck, bitter mouth, palpitation, increased perspiration, poor night rest, difficulty in falling asleep, early awakening, red tongue, white fur, and deep and tight pulse.

According to Western medicine, the patient was diagnosed with hyperthyroidism, whereas based on TCM, the diagnosis was gall disease (hyperactivity of fire in liver meridian), which was treated with the following methods: clearing liver and purging fire, and regulating qi and resolving phlegm. The patient was prescribed the medicinal Longdan Xiegan Decoction, consisting of longdancao $6 \mathrm{~g}$, stir-fried gardenia $6 \mathrm{~g}$, bupleurum 10 g, Alisma 10 g, Scutellaria 6 g, Shengdi 15 g, Danshen 20 g, Baishao 15 g, Prunella vulgaris $10 \mathrm{~g}$, and peony bark. The patient was given 7 doses and was asked to take 1 dose daily. The decoction was taken warm after breakfast and dinner. The patient was followed up for 1 month and was asked to relax and eat regularly.

On September 24, 2020, after taking the medicine, the feeling of choking and distension was obviously alleviated. In the morning, there was occasional bitter mouth and fatigue, which was significantly improved compared with before. Occasionally, she was flustered, without obvious sweating. She had general appetite and sleep, with red tongue tip, white and thick fur, and deep pulse. The patient was instructed to orally take $2.5 \mathrm{mg}$ of methimazole tablets every other day, and then treated according to the original intention. Based on the above prescription, Rhizoma alismatis was removed. The patient was also another prescription containing Cortex Moutan, bamboo leaf 6 g, Amomum villosum 6 g, houxia, Yujin 15 g, Zhigancao 6 g, Xuanshen $20 \mathrm{~g}$, and Danshen $20 \mathrm{~g}$. The patient was given 12 doses, and was instructed to take 1 dose daily. The medicine was taken warm after breakfast and dinner. The patients were followed up for 1 month.

On October 25, 2020, after taking the medicine, the feeling of suffocation and distension disappeared, the bitter mouth disappeared, and there was no obvious fatigue. The tongue was light red, the moss was thin and white, and the pulse is stringy. The patient was asked to stop using methimazole tablets, Shugan Jieyu, and Jianpi Yangxue, but to take Fang Xuan Xiaoyao Powder that contains Chaihu 15 g, Danggui 12 g, Fuling 15 g, Baishao 15 g, Baizhu 15 g, ginger 6 g, mint 3 g, houxia, licorice 6 g, fried gardenia $6 \mathrm{~g}$, peony $15 \mathrm{~g}$, Prunella $15 \mathrm{~g}$, and ginger Pinellia $10 \mathrm{~g}$. 
The patient is a middle-aged female, who has been suffering from many trivial matters, such as rough emotion of liver $q i$, stagnation of $q i$ and phlegm coagulation, swelling in front of neck, stagnation of $q i$ for a long time, transforming into fire, and inversion of liver qi. Caused by depressed liver, which is vital for women, it can be seen that menstruation is irregular, $q i$ stagnation and blood stasis happen in the body, and it is noteworthy that long-term illness is characterized by deficiency of both qi and yin. Professor Pei used the method of soothing liver, nourishing yin and clearing liver fire as the treatment. She chose Longdan Xiegan Decoction plus Salvia miltiorrhiza and Mudanpi to promote blood circulation and remove blood stasis, Radix Paeoniae Alba to soften liver, Prunella vulgaris to disperse, Amomum villosum to remove dampness and stomach, Radix Scrophulariae and Salvia miltiorrhiza to disperse, and herbs to clear liver fire, regulate $q i$ and resolve phlegm. Because of the patient's long-term emotional distress, she was treated with soothing the liver, invigorating the spleen and nourishing the blood after clearing the liver fire in the third diagnosis.

\section{Summary}

Professor Ruixia Pei uses the "harmony" method to treat hyperthyroidism from the liver using not only symptomatic treatment, but also pay close attention to the development of pathogenesis. In clinical diagnosis and treatment, we should pay attention to individual differences, regulate patients' emotions, adjust their eating habits, and put forward consolidation treatment in the initial recovery of the disease.

\section{Disclosure statement}

The authors declare that there is no conflict of interest.

\section{References}

[1] Shuai Y, Zhang X, Li H, 2019, Li Huilin's Experience in Treating Hyperthyroidism with Jiakang Yangyin Formula. Guangzhou University of Traditional Chinese Medicine, 36(03): 1423-427.

[2] Wei X, 2020, Review of Methimazole in the Treatment of Hyperthyroidism. Chinese Medical Guideline, 18(14): 29-30.

[3] Dou D, Li Z, 2019, Li Zhongnan's Experience in Treating Hyperthyroidism with Syndrome Differentiation. Journal of Shaanxi University of Chinese Medicine, 42(01).

[4] Wang Y, Liu H, 2019, Liu Huai Zhen's Experience in Treating Hyperthyroidism. Clinical Journal of Traditional Chinese Medicine, 31(12): 2229-2232.

[5] Wang D, Feng M, 2019, Research Progress of Hyperthyroidism in Traditional Chinese Medicine. World Latest Medical Information Abstracts, 19(66).

[6] Xu X, Xu T, 2016, Gongping's Experience in Treating Hyperthyroidism. Sichuan Traditional Chinese Medicine, 34(9): 12-14.

[7] Han X, He Z, 2018, Professor He Ze's Clinical Experience in Treating Hyperthyroidism from Liver. Modern Distance Education of Traditional Chinese Medicine in China, 16(7). 